\title{
Clinical Score and Transcript Abundance Patterns Identify Kawasaki Disease Patients Who May Benefit From Addition of Methylprednisolone
}

\author{
SHOHEI OGATA, YOSHIHITO OGIHARA, KEIKO NOMOTO, KAZUMASA AKIYAMA, YAYOI NAKAHATA, KAYOKO SATO, \\ KATSUNORI MINOURA, KENICHI KOKUBO, HIROSUKE KOBAYASHI, AND MASAHIRO ISHII

\begin{abstract}
Department of Pediatrics [S.O., Y.O., K.N., K.A., Y.N., M.I.], Kitasato University School of Medicine, Sagamihara, Kanagawa 228-0855, Japan; Department of Medical Engineering and Technology [K.K., H.K.], Kitasato University School of Allied Health Sciences, Sagamihara, Kanagawa 228-0855, Japan; Department of Pediatrics [K.S., K.M.], Ebina General Hospital, Ebina, Kanagawa 243-0433, Japan
\end{abstract}

\begin{abstract}
Intravenous immunoglobulin (IVIG) treatmentresistant patients are high risk of developing coronary artery lesions with Kawasaki disease. The IVIG-responsive (Group A; $n=6$ ) and IVIG-resistant patients (Group B) were predicted before starting the initial treatment using the Egami scoring system and randomly allocated as a single-IVIG treatment group (group B1; $n=6$ ) or as a IVIG-plus-methylprednisolone (IVMP) combined therapy group (group B2; $n=5$ ). We investigated the transcript abundance in the leukocytes of those patients using a microarray analysis. Five patients in group A and one patient in group B1 responded to initial IVIG treatment. All group B2 patients responded to IVIG-plus-IVMP combined therapy. Before performing these treatments, those transcripts related to IVIG resistance and to the development of coronary artery lesions, such as IL1R, IL18R, oncostatin M, suppressor of cytokine signaling-3, S100A12 protein, carcinoembryonic antigenrelated cell adhesion molecule-1, matrix metallopeptidase-9, and polycythemia rubra vera-1, were more abundant in group B patients in comparison with group A patients. Moreover, those transcripts in group B2 patients were more profoundly and broadly suppressed than group B1 patients after treatment. This study elucidated the molecular mechanism of the effectiveness of IVIG-plus-IVMP combined therapy. (Pediatr Res 66: 577-584, 2009)
\end{abstract}

$\mathrm{K}^{\mathrm{a}}$ awasaki disease (KD) is an acute febrile disorder characterized by systemic vasculitis that primarily affects infant and children (1). Treatment with high-dose intravenous immunoglobulin (IVIG) and aspirin results in a resolution of fever in most patients with KD while also significantly reducing the incidence of coronary artery lesions (CALs) (2). However, approximately 15 to $20 \%$ of patients with KD have IVIG resistance, even though standard high-dose IVIG therapy was appropriately initiated (3). In these patients, CALs frequently developed, despite the use of additional treatments, including further IVIG and steroids, thus indicating that current alternative treatments are ineffective after the failure of

Received March 7, 2009; accepted July 17, 2009

Correspondence: Masahiro Ishii, MD, PhD, Department of Pediatrics, Kitasato University School of Medicine, 1-15-1, Kitasato, Sagamihara, Kanagawa 228-0855, Japan; e-mail: ishiim@med.kitasato-u.ac.jp

This work was supported by a Grant-in-aid for Scientific Research (C: 9022950) from the Ministry of Education, Culture, Sports, Science and Technology; a Parents' Association Grant at Kitasato University School of Medicine; a Grant-in-aid from the Morinaga Foundation for Health and Nutrition; and a Grant-in-aid from the Kawasaki Disease Research Center in Japan. the initial IVIG treatment (4-6). In other words, an earlier and more effective primary therapy is required for IVIG-resistant patients (7-11). Steroid therapy has been reconsidered as a therapeutic strategy to treat KD (11-15). A novel therapeutic approach may not be necessary in all cases of KD because the appropriate dose of IVIG and aspirin yields a good response in 80 to $85 \%$ of patients. Therefore, we predicted IVIG-resistant patients before starting the initial treatment using the Egami score. The Egami scoring system identifies age, days of illness, platelet count, C-reactive protein, and alanine aminotransferase (ALT) to predict IVIG-resistant patients before treatment (cutoff: 3 points; $78 \%$ sensitivity and $76 \%$ specificity) (9). The Egami score is highly sensitive and specific in Japanese patients with KD but is not highly sensitive or specific in the mixed ethnic US population (8). This study randomly devised IVIG-plus-methylprednisolone (IVMP) combined therapy or single-IVIG treatment as a primary treatment in Japanese patients with prediction of IVIG resistant in a prospective study and investigated the biologic mechanism of those treatments for prediction of IVIGresistant patients using a microarray analysis.

\section{PATIENTS AND METHODS}

Patients and study protocol. Sixty-nine patients with KD had been admitted to Kitasato University Hospital or Ebina General Hospital from 2006 to 2008. All patients were Japanese and fulfilled the criteria for the guidelines established by the Kawasaki Disease Research Committee of Japan (http:// www.kawasaki-disease.org/tebiki/tebiki.html) (16). The diagnosis and evaluation of CALs were carried out using two-dimensional echocardiography before the initial treatment, at 36 to $48 \mathrm{~h}$ after treatment, and at 1 mo after treatment. The presence of CALs was diagnosed according to the Japanese Ministry of Health criteria. IVIG-responsive patients were defined as those who exhibited resolution of fever $\left(<37.5^{\circ} \mathrm{C}\right)$ within 36 to $48 \mathrm{~h}$ after receiving the initial treatment $(13,15)$.

Figure 1 shows the study protocol. Eleven of 69 patients with KD (15.9\%) were predicted IVIG-resistant patients by Egami score (group B). These patients were randomly assigned to the single-IVIG treatment group (group $\mathrm{B} 1 ; n=6$ ) and IVIG-plus-IVMP combined therapy group (group B2; $n=5$ ), using a table of random digits. The remaining 58 patients were predicted IVIG-responsive patients by Egami score. The six of 58 patients who were predicted IVIG responders by Egami score were randomly selected as group A. Groups A and B1 patients were administered single-IVIG treatment $(2.0 \mathrm{~g} / \mathrm{kg}$ for

\footnotetext{
Abbreviations: CALs, coronary artery lesions; HP, haptoglobin; IVIG, intravenous immunoglobulin; IVMP, IVIG-plus-methylprednisolone; KD, Kawasaki disease; PRV-1, polycythemia rubra vera-1
} 


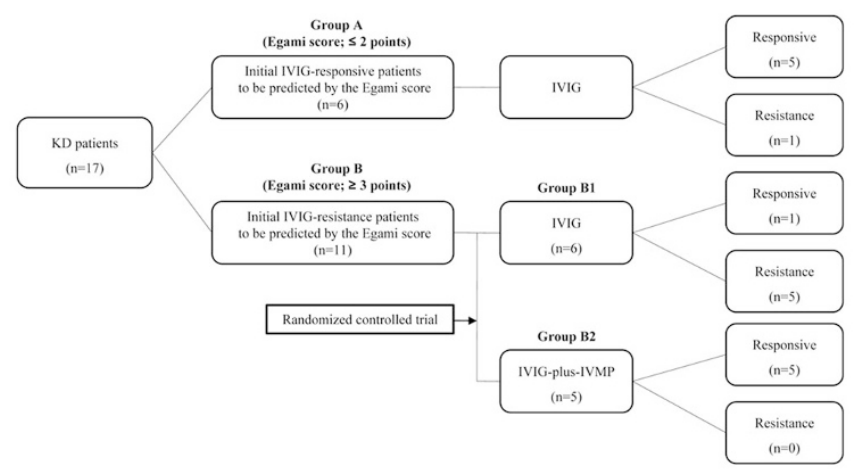

Figure 1. Study protocol and treatment results. The subjects were divided into the following groups: predicted to be IVIG-responsive patients (group A) and predicted to be IVIG-resistant patients (group B). Group A patients were administered single-IVIG ( $2.0 \mathrm{~g} / \mathrm{kg}$ for $1 \mathrm{~d})$. Group B patients were randomly assigned to receive either single-IVIG $(2.0 \mathrm{~g} / \mathrm{kg}$ for $1 \mathrm{~d}$, group B1) or IVIG-plus-IVMP combined therapy (IVMP: $30 \mathrm{mg} / \mathrm{kg}$ for $2 \mathrm{~h}$, group B2).

1 d). Group B2 patients received IVMP therapy, $30 \mathrm{mg} / \mathrm{kg}$ during $2 \mathrm{~h}$ before receiving IVIG treatment $(2.0 \mathrm{~g} / \mathrm{kg}$ for $1 \mathrm{~d})$. All patients received aspirin (30 $\mathrm{mg} / \mathrm{kg}$ per day) during the acute stage of illness. The dose of aspirin decreased to $5 \mathrm{mg} / \mathrm{kg}$ per day after defervescence. Venous blood was drawn from each patient before treatment and at 36-48 h after treatment. Parental informed consent was obtained for each child enrolled in this study. The Ethical Committee of Kitasato University and Ebina General Hospital approved this study.

RNA isolation, cDNA preparation, and hybridization. Total RNA was isolated from $2.5 \mathrm{~mL}$ of whole blood using a PAXgene tube, according to the manufacturer's recommended protocol (Paxgene Blood RNA Kit, PreanalytiX, QIAGEN, Valencia, CA). Next, $\alpha$-globin and $\beta$-globin mRNA were removed from the total RNA using the globin reduction method according to the manufacturer's recommended protocol (Affymetrix, Santa Clara, CA). The synthesis of cRNA and hybridization were performed using the one-cycle target labeling and control reagents according to the manufacturer's recommended protocol (Affymetrix) and methods described in previous reports $(17,18)$. Briefly, $2 \mu \mathrm{g}$ samples of total RNA from which globin mRNA had been removed were individually converted to double-stranded cDNA (dscDNA) using the one-cycle cDNA synthesis kit (Affymetrix). The cDNA was then subjected to in vitro transcription in the presence of biotinylated uridine triphosphate, using an IVT labeling kit (Affymetrix). Qualitative analyses of the isolated total RNA and synthesized cRNA were conducted by electropherogram (Experion; Bio-Rad Laboratories, Hercules, CA). The biotinylated cRNA was fragmented and hybridized for $16 \mathrm{~h}$ at $45^{\circ} \mathrm{C}$ with the Human Genome U133 plus 2.0 array (Affymetrix), which contains the oligonucleotide probe set for 54,675 full-length transcripts and expressed sequence tags. The arrays were washed, stained with streptavidin-phycoerythrin, and scanned using an Affymetrix Model Fluidics Station 450 and GeneChip Scanner 3000 (Affymetrix). The fluorescence intensity of each probe was quantified using the computer program GeneChip operating software, GCOS version 1.4 (Affymetrix). Each microarray was subjected to a standard quality control evaluation; the percentage of probe sets reliably detecting (present flag) was between 40 and $60 \%$, and the $3^{\prime} / 5^{\prime}$ ratio of the $G A P D H$ was $<3$. All background intensities and noise factors were within the range of 31.37 to 55.72 and 1.3 to 2.35 , respectively. The results of the GeneChip analysis can be found on GEO (http://www.ncbi.nlm.nih.gov/geo).

Microarray data analysis. The expression analysis file created from each chip (sample) was imported into GeneSpring GX version 7.3 software (Agilent Technologies, Santa Clara, CA) for further data characterization. Each array was normalized (mean centered) to the median intensity array. To minimize the influence of background noise, only probes reliably detected in at least two out of all the samples were included in the analysis. A one-way ANOVA analysis and gene ontology analysis using Fisher's exact test and the unsupervised hierarchical clustering analysis using Pearson's correlation were performed using the GeneSpring GX version 7.3 software package.

Quantitative real-time PCR. cDNA was generated from the total RNA after exclusion globin mRNA samples using a QuantiTect Reverse Transcription System kit (QIAGEN) using random primers and Oligo-dT primers. The PCR primers for CD177 (polycythemia rubra vera-1; prv-1), haptoglobin $(h p)$, carcinoembryonic antigen-related cell adhesion molecule 1 (ceacam1), suppressor of cytokine signaling 3 (socs3), and oncostatin-m (osm) were designed based on the GeneBank sequence, and the PCR primers were designed using the Primer 3 software program (http://primer3.sourceforge.net/). The primer sequences were as follows: prv-1 forward primer, 5'-GGCCCAACCTTCCAGCTT-3'; prv-1 reverse primer, 5'-CTTCTCACGCGCAGAGAAG-3'; $h p$ forward primer, 5'-CTGGAGGAGGACACCTGGTA-3'; $h p$ reverse primer, 5'-TGGTCTTCTGAACCCAGTCC-3'; ceacam 1 forward primer, 5'-ACCCACCTGCACAGTACTCC-3'; ceacam1 reverse primer, 5'-TCGTCTTGACTGTGGTCCTG3'; socs 3 forward primer, 5'-GCCACCTACTGAACCCTCCT-3'; socs 3 reverse primer, 5'-AACACCAGGGGGATCTTCTC-3'; osm forward primer, 5'TACCATCGCTTCATGCACTC-3'; and osm reverse primer, 5'-GGAGGAGGTAGAGGGGTCTG-3'. RT-PCR was conducted using the Chromo 4 System (Bio-Rad laboratories) in a $25-\mu \mathrm{L}$ reaction mixture containing $12.5 \mu \mathrm{L}$ of iQ SYBR Green SuperMix (Bio-Rad Laboratories), $1 \mu \mathrm{L}$ of a mixture of forward and reverse primer $(10 \mathrm{pmol} / \mu \mathrm{L})$, and $11.5 \mu \mathrm{L}$ of cDNA diluted in RNase-free $\mathrm{H}_{2} \mathrm{O}$. Samples were preincubated for $3 \mathrm{~min}$ at $95^{\circ} \mathrm{C}$, and then were subjected to 40 cycles of amplification at $94^{\circ} \mathrm{C}$ for $15 \mathrm{~s}$ for denaturing and at $60^{\circ} \mathrm{C}$ for $30 \mathrm{~s}$ for annealing and $72^{\circ} \mathrm{C}$ for extension. The expression levels of each target cDNA relative to GAPDH were calculated using a comparative $\mathrm{Ct}$ method, and the levels were determined for each sample (19).

Statistical analysis. Analyses of clinical variables were conducted using the SPSS statistical software package, version 15 (SPSS Japan, Tokyo). Summary statistics (median, SD, and percentages) was calculated to describe the subject characteristics. For the data regarding characteristics of the study population, the laboratory and the RT-PCR data, Kruskal-Wallis test nonparametric ANOVA and Mann-Whitney $U$ test were used to compare samples obtained from patients in each treatment group. Wilcoxon's signed rank test was used to compare the results before the initial treatment to those obtained after the initial treatment. Regarding the data of transcripts abundance, one-factor ANOVA used to compare each of the groups. The $p$ values were corrected by use of the Bonferroni/Dunn $F$ test as a post hoc test, if not otherwise indicated. A value of $p<0.05$ was considered to be statistically significant.

\section{RESULTS}

Characteristics and clinical outcome. Five patients in group A $(n=6)$ showed a response to IVIG treatment $(83.3 \%)$. One patient in group B1 $(n=6)$ was responsive to IVIG treatment $(16.7 \%)$, and all patients in group B2 $(n=5)$ responded to the IVIG-plus-IVMP combination therapy (100\%; Fig. 1). Four patients exhibited transient coronary dilation in the acute phase of illness or 36 to $48 \mathrm{~h}$ after the initial treatment (group A: 1 patient; group B1: 3 patients). However, none of the patients developed CALs at 1 mo after onset of the disease. None of the patients experienced any side effects of IVMP therapy (e.g., hypothermia, bradycardia, and hypertension). Tables $1-3$ show the demographic and characteristics of the study population. The lymphocyte count and the aspartate aminotransferase and ALT levels were significantly different between groups A and B before the initial treatment. No significant differences between patient demographics and characteristics were observed between groups B1 and B2 before initial treatment. However, significant differences were observed posttreatment in the lymphocyte counts and ALT levels of group A versus groups B1 or B2 patients.

Profiles of transcript abundance in patients that stratified by the Egami score. Using an unsupervised hierarchical clustering approach, clustering analysis of transcript abundance pattern was conducted in the pretreatment samples from groups A, B1, and B2 patients (Fig. 2A). The clustering analysis used 7803 of 54,675 transcripts that showed significantly altered expression levels among the three groups (oneway ANOVA; $p<0.05$ ). The transcript abundance pattern in the pretreatment samples from group B1 patients showed good similarity to that of group B2 patients $\left(r^{2}=0.59\right)$. In contrast, no similarity between the transcript abundance pattern of groups A and B (group B included both groups B1 and B2) patients was observed $\left(r^{2}=0.15\right)$. 
Table 1. Demographic data of the subjects before treatment

\begin{tabular}{|c|c|c|c|c|}
\hline & Group A & Group B1 & Group B2 & $p$ \\
\hline Sex (male, female) & $\mathrm{m} 3, \mathrm{f} 3$ & $\mathrm{~m} \mathrm{4,} \mathrm{f} 2$ & $\mathrm{~m} 3, \mathrm{f} 2$ & \\
\hline Age $(\mathrm{mo})$ & $31 \pm 24.1$ & $44.0 \pm 23.9$ & $26.0 \pm 24.0$ & 0.88 \\
\hline Illness days of initial treatment $(\mathrm{d})$ & $5.0 \pm 0.9$ & $3.5 \pm 1.0$ & $4.0 \pm 1.1$ & 0.11 \\
\hline WBC count $\left(/ \mathrm{mm}^{3}\right)$ & $14,450.0 \pm 7233.2$ & $11,000.0 \pm 6343.6$ & $12,000.0 \pm 3589.6$ & 0.58 \\
\hline Neutrophilic count $\left(/ \mathrm{mm}^{3}\right)$ & $9516.0 \pm 6140.1$ & $8202.6 \pm 5933.9$ & $8996.4 \pm 1749.4$ & 0.91 \\
\hline Lymphocyte count $\left(/ \mathrm{mm}^{3}\right)$ & $3664.0 \pm 2285.6$ & $1725.0 \pm 362.0$ & $671.5 \pm 2408.9$ & 0.07 \\
\hline Monocytic count $\left(/ \mathrm{mm}^{3}\right)$ & $609.8 \pm 359.9$ & $633.6 \pm 402.9$ & $552.0 \pm 172.5$ & 0.74 \\
\hline Hemoglobin $(\mathrm{g} / \mathrm{dL})$ & $11.3 \pm 1.0$ & $11.5 \pm 0.7$ & $12.1 \pm 1.0$ & 0.59 \\
\hline Platelet count $\left(\times 10^{4} / \mathrm{mm}^{3}\right)$ & $34.1 \pm 11.9$ & $27.9 \pm 9.6$ & $27.0 \pm 10.6$ & 0.45 \\
\hline AST (IU/L) & $47.5 \pm 17.0 \dagger \dagger$ & $116.5 \pm 324.5 \dagger$ & $155.0 \pm 108.5$ & $0.02 *$ \\
\hline ALT (IU/L) & $12.0 \pm 12.3 \dagger \dagger$ & $221.0 \pm 122.2 \dagger$ & $242.0 \pm 92.8 \ddagger$ & $0.01 *$ \\
\hline Albumin $(\mathrm{g} / \mathrm{dL})$ & $3.7 \pm 0.2$ & $3.7 \pm 0.4$ & $3.9 \pm 0.2$ & 0.46 \\
\hline Sodium $(\mathrm{mEq} / \mathrm{L})$ & $133.0 \pm 2.1$ & $131.0 \pm 3.7$ & $131.0 \pm 3.7$ & 0.38 \\
\hline $\mathrm{CRP}(\mathrm{mg} / \mathrm{dL})$ & $9.3 \pm 7.0$ & $9.4 \pm 4.5$ & $6.2 \pm 5.0$ & 0.33 \\
\hline $\operatorname{ESR}(\mathrm{mm} / \mathrm{h})$ & $83.0 \pm 12.3$ & $71.5 \pm 13.1$ & $68.0 \pm 25.9$ & 0.27 \\
\hline $\operatorname{IgG}(\mathrm{mg} / \mathrm{dL})$ & $598.0 \pm 245.1$ & $623.5 .0 \pm 202.5$ & $773.0 \pm 73.6$ & 0.30 \\
\hline
\end{tabular}

Group A: Egami score $\leq 2$ point, group B1: $\geq 3$ points; IVIG treatments group, group B2: $\geq 3$ points; IVIG-plus-IVMP combined therapy group.

A value of $p<0.05$ was considered to be statistically significant.

$* p$ value calculation by Kruskal-Wallis test.

$\dagger \neq p$ value calculation by Bonferroni test.

Table 2. Demographic data of the subjects after treatment

\begin{tabular}{|c|c|c|c|c|}
\hline & Group A & Group B1 & Group B2 & $p$ \\
\hline WBC count $\left(/ \mathrm{mm}^{3}\right)$ & $8100.0 \pm 3042.1$ & $11,900.0 \pm 4837.1$ & $9600.0 \pm 2717.9$ & 0.32 \\
\hline Neutrophic count $\left(/ \mathrm{mm}^{3}\right)$ & $3180.0 \pm 2171.8$ & $9302.3 \pm 4896.1$ & $3273.2 \pm 1213.9$ & 0.21 \\
\hline Lymphocyte count $\left(/ \mathrm{mm}^{3}\right)$ & $3720.0 \pm 1128.0 \dagger$ & $2044.8 \pm 837.2 \dagger$ & $4294.6 \pm 2095.4$ & $0.02 *$ \\
\hline Monocytic count $\left(/ \mathrm{mm}^{3}\right)$ & $569.4 \pm 167.4$ & $606.0 \pm 268.6$ & $548.8 \pm 378.4$ & 0.87 \\
\hline Hemoglobin $(\mathrm{g} / \mathrm{dL})$ & $11.5 \pm 1.6$ & $11.0 \pm 1.0$ & $11.5 \pm 0.76$ & 0.28 \\
\hline Platelet count $\left(\times 10^{4} / \mathrm{mm}^{3}\right)$ & $40.3 \pm 15.0$ & $34.3 \pm 11.1$ & $26.5 \pm 14.3$ & 0.52 \\
\hline AST (IU/L) & $35.5 \pm 16.8$ & $30.5 \pm 3.4$ & $37.0 \pm 14.7$ & 0.32 \\
\hline ALT (IU/L) & $12.5 \pm 20.2 \dagger$ & $48.5 \pm 19.2$ & $70.0 \pm 38.7 \dagger$ & $0.02 *$ \\
\hline Albumin $(\mathrm{g} / \mathrm{dL})$ & $3.4 \pm 0.3$ & $2.9 \pm 0.8$ & $3.2 \pm 0.22$ & 0.29 \\
\hline Sodium $(\mathrm{mEq} / \mathrm{L})$ & $136.0 \pm 2.3$ & $134.5 \pm 4.6$ & $136.0 \pm 1.8$ & 0.65 \\
\hline $\mathrm{CRP}(\mathrm{mg} / \mathrm{dL})$ & $2.9 \pm 2.3$ & $5.7 \pm 5.0$ & $1.4 \pm 0.96$ & 0.06 \\
\hline $\operatorname{ESR}(\mathrm{mm} / \mathrm{h})$ & $71.0 \pm 20.6$ & $70.5 \pm 30.3$ & $18.0 \pm 73.0$ & 0.96 \\
\hline $\operatorname{IgG}(\mathrm{mg} / \mathrm{dL})$ & $2466.0 \pm 465.9$ & $2715.5 \pm 513.0$ & $2714.0 \pm 150.1$ & 0.58 \\
\hline
\end{tabular}

A value of $p<0.05$ was considered to be statistically significant.

$* p$ value calculation by Kruskal-Wallis test.

$\dagger+p$ value calculation by Bonferroni test.

We next examined to transcripts significantly differenced expression level between groups A and B patients, between IVIG-responder and IVIG-resistant patients, respectively. In pretreatment samples, 8917 of 54,675 transcripts showed significantly differenced expression levels between groups A $(n=6)$ and $\mathrm{B}(n=11)$, as determined by a one-way ANOVA $(p<0.05)$. Moreover, 6316 of 8917 transcripts were not only groups A and B but also significantly differenced expression levels between IVIG-responsive patients $(n=6)$ and IVIGresistant patients ( $n=6$; IVIG-resistant patient in group A: one patient, in group B1: five patients) based on a Venn diagram analysis. In a comparison of groups A and B using 6316 transcripts, 432 transcripts were more than double regulation and 179 transcripts were less than half regulation in group B. Table 4 shows the profile of the most up-regulated transcripts in group B.

Profiles of transcript abundance in single-IVIG and IVIG-plus-IVMP combined therapy. A hierarchical cluster analysis after treatments showed that the transcript abundance pattern of samples in group B2 had strong similarities to samples from IVIG responders $\left(r^{2}=0.66\right.$; Fig. $\left.2 B\right)$. The transcript abundance pattern after treatment of group B1 patients was not similar to that of group B2 patients $\left(r^{2}=0.05\right)$.

Table 5 provides the transcripts after treatment were less abundant than those before treatment. Each group included transcripts for $P R V-1, I L 18 R 1$, SOCS3, OSM, complement component $3 b / 4 b$ receptor 1 (CR1), HP, ILIR2, and $C E A C A M 1$. The transcripts of the group $\mathrm{B} 2$ patients were less abundant than those of the groups A and B1 patients.

In addition, the number of transcripts that were $\geq 2 \mathrm{SD}$ down-regulated between pre- and posttreatments in each group were group A; $1906 \pm 545.5$ transcripts, group B1: $1085.7 \pm 805.3$ transcripts, and group B2: $2982.0 \pm 471.5$ transcripts. There were significantly more regulating transcripts in group B2 with a $\geq 2 \mathrm{SD}$ than in either groups $\mathrm{A}$ and B1 $(p<0.001)$.

The characteristics of the down-regulated transcripts after treatment in groups B1 and B2. The distribution of those transcripts that $\geq 2$ SD varied between pre- and posttreatment in groups B1, B2, and IVIG responders using a Venn diagram 
Table 3. Comparison of samples between before and after the initial treatment

\begin{tabular}{|c|c|c|c|c|c|c|c|c|c|}
\hline & \multicolumn{3}{|c|}{ Group A } & \multicolumn{3}{|c|}{ Group B1 } & \multicolumn{3}{|c|}{ Group B2 } \\
\hline & Pre & Post & $p$ & Pre & Post & $p$ & Pre & Post & $p$ \\
\hline $\begin{array}{l}\text { WBC count } \\
\left(/ \mathrm{mm}^{3}\right)\end{array}$ & $14,450.0 \pm 7233.2$ & $8100.0 \pm 3042.1$ & $0.03 *$ & $11,000.0 \pm 6343.6$ & $11,900.0 \pm 4837.1$ & 0.35 & $12,000.0 \pm 3589.6$ & $9600.0 \pm 2717.9$ & $0.04 *$ \\
\hline $\begin{array}{l}\text { Neutrophil count } \\
\left(/ / \mathrm{mm}^{3}\right)\end{array}$ & $9516.0 \pm 6140.1$ & $3180.0 \pm 2171.8$ & $0.03 *$ & $8202.6 \pm 5933.9$ & $9302.3 \pm 4896.1$ & 0.35 & $8996.4 \pm 1749.4$ & $3273.2 \pm 1213.9$ & $0.04 *$ \\
\hline $\begin{array}{l}\text { Lymphocyte count } \\
\qquad\left(/ \mathrm{mm}^{3}\right)\end{array}$ & $3664.0 \pm 2285.6$ & $3720.0 \pm 1128.0$ & 0.92 & $1725.0 \pm 362.0$ & $2044.8 \pm 837.2$ & 0.35 & $671.5 \pm 2408.9$ & $4294.6 \pm 2095.4$ & 0.08 \\
\hline $\begin{array}{l}\text { Monocyte count } \\
\left(/ \mathrm{mm}^{3}\right)\end{array}$ & $609.8 \pm 359.9$ & $569.4 \pm 167.4$ & 0.60 & $633.6 \pm 402.9$ & $606.0 \pm 268.6$ & 0.35 & $552.0 \pm 172.5$ & $548.8 \pm 378.4$ & 0.50 \\
\hline $\begin{array}{l}\text { Hemoglobin } \\
\qquad(\mathrm{g} / \mathrm{dL})\end{array}$ & $11.3 \pm 1.0$ & $11.5 \pm 1.6$ & 0.47 & $11.5 \pm 0.7$ & $11.0 \pm 1.0$ & 0.05 & $12.1 \pm 1.0$ & $11.5 \pm 0.76$ & 0.59 \\
\hline $\begin{array}{l}\text { Platelet count } \\
\qquad\left(\times 10^{4} / \mathrm{mm}^{3}\right)\end{array}$ & $34.1 \pm 11.9$ & $40.3 \pm 15.0$ & 0.05 & $27.9 \pm 9.6$ & $34.3 \pm 11.1$ & 0.60 & $27.0 \pm 10.6$ & $26.5 \pm 14.3$ & 0.23 \\
\hline AST (IU/L) & $47.5 \pm 17.0$ & $35.5 \pm 16.8$ & 0.89 & $116.5 \pm 324.5$ & $30.5 \pm 3.4$ & $0.03 *$ & $155.0 \pm 108.5$ & $37.0 \pm 14.7$ & $0.04 *$ \\
\hline ALT (IU/L) & $12.0 \pm 12.3$ & $12.5 \pm 20.2$ & 0.59 & $221.0 \pm 122.2$ & $48.5 \pm 19.2$ & $0.03 *$ & $242.0 \pm 92.8$ & $70.0 \pm 38.7$ & $0.04 *$ \\
\hline Albumin (g/ dL) & $3.7 \pm 0.2$ & $3.4 \pm 0.3$ & $0.03 *$ & $3.7 \pm 0.4$ & $2.9 \pm 0.8$ & $0.03 *$ & $3.9 \pm 0.2$ & $3.2 \pm 0.22$ & $0.04 *$ \\
\hline Sodium $(\mathrm{mEq} / \mathrm{L})$ & $133.0 \pm 2.1$ & $136.0 \pm 2.3$ & $0.02 *$ & $131.0 \pm 3.7$ & $134.5 \pm 4.6$ & 0.24 & $131.0 \pm 3.7$ & $136.0 \pm 1.8$ & $0.04 *$ \\
\hline $\mathrm{CRP}(\mathrm{mg} / \mathrm{dL})$ & $9.3 \pm 7.0$ & $2.9 \pm 2.3$ & $0.03 *$ & $9.4 \pm 4.5$ & $5.7 \pm 5.0$ & 0.25 & $6.2 \pm 5.0$ & $1.4 \pm 0.96$ & $0.04 *$ \\
\hline $\operatorname{ESR}(\mathrm{mm} / \mathrm{h})$ & $83.0 \pm 12.3$ & $71.0 \pm 20.6$ & 0.35 & $71.5 \pm 13.1$ & $70.5 \pm 30.3$ & 0.92 & $68.0 \pm 25.9$ & $18.0 \pm 73.0$ & 0.59 \\
\hline $\operatorname{IgG}(\mathrm{mg} / \mathrm{dL})$ & $598.0 \pm 245.1$ & $2466.0 \pm 465.9$ & $0.03 *$ & $623.5 .0 \pm 202.5$ & $2715.5 \pm 513.0$ & $0.03 *$ & $773.0 \pm 73.6$ & $2714.0 \pm 150.1$ & $0.04 *$ \\
\hline
\end{tabular}

A value of $p<0.05$ was considered to be statistically significant.

* $p$ value calculation by Wilcoxon signed rank test.

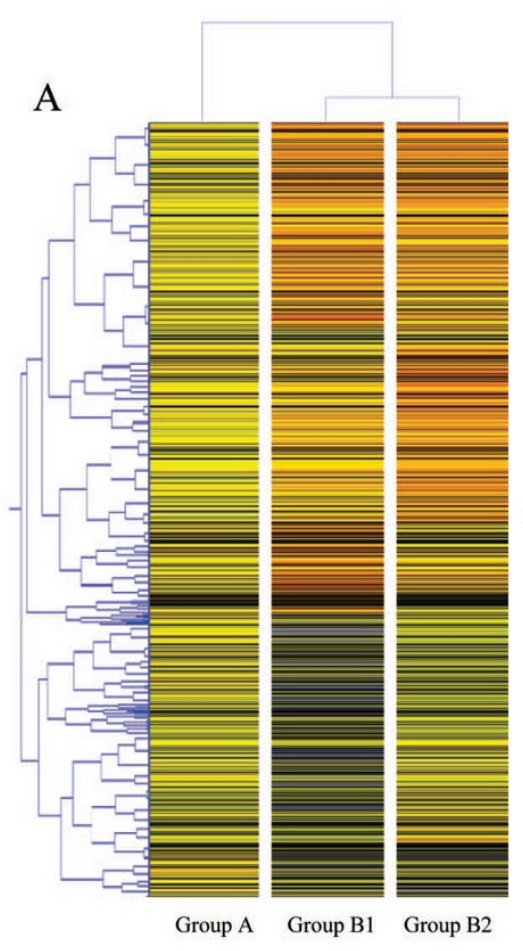

B

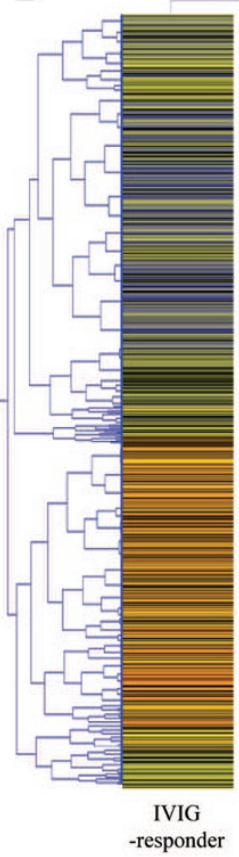

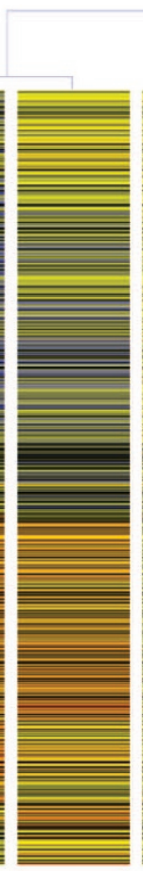
Group B2

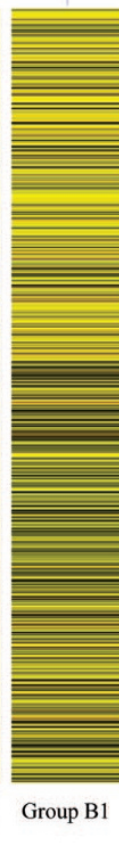

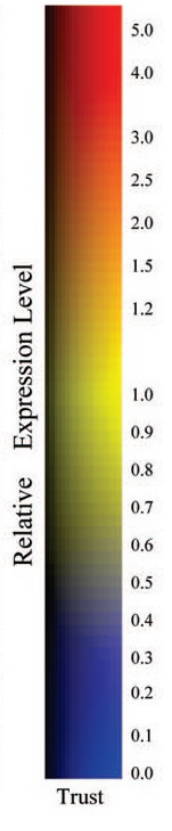

Figure 2. Unsupervised hierarchical clustergram analysis using Pearson's correlation. $(A)$ An analysis of the pretreatment samples. $(B)$ An analysis of the posttreatment samples. The transcript abundance pattern of group B2 was more like that of group B1 than group A in pretreatment samples (groups A vs B: $r^{2}=0.15$, groups $\mathrm{B} 1$ vs. B2: $r^{2}=0.56$ ). The transcript abundance pattern in posttreatment samples showed strong similarities between the IVIG-responder group and group B2 (IVIG responder group $v s$ group B2: $r^{2}=0.66$, groups $\left.\mathrm{B} 1 v s \mathrm{~B} 2 ; r^{2}=0.05\right)$. is shown in Figure 3. In this distribution analysis, 707 transcripts were $\geq 2$ SD less abundant in IVIG responders and group B2 patients, 945 transcripts in only group B2 patients, and 34 transcripts in only group B1 patients. The Gene Ontology browser was used to analyze the 707 transcripts that were less abundant in IVIG-responder group and group B2, and 945 transcripts that were less abundant in only group B2. Table 6 shows the categories of biologic processes for theses transcripts, respectively. The 707 transcripts had transcripts related to the positive regulation of the $\mathrm{I}-\kappa \mathrm{B}$ kinase/NF- $\kappa \mathrm{B}$ cascade, immune response and cytokine production, neutrophil activation, TNF $\alpha$ synthesis, and regulation of IL $1 \beta$ in the category of immune response (Fisher's exact test; $p<0.001$ ). The 945 transcripts had transcripts related to apoptosis, inositol phosphate dephosphorylation, and B-cell activation, natural killer cell differentiation and $\mathrm{T}$-cell regulation in the category of immune response $(p<0.001)$. The 34 transcripts with $\geq 2$ SD down-regulated in only group B1 patients included transcripts such as polycomb group ring finger-2 (PCGF2), platelet-derived growth factor alpha polypeptide 
Table 4. Up-regulated transcripts in the groups A patients before treatment

\begin{tabular}{|c|c|c|c|}
\hline Probe ID & Gene symbol & Gene name & Fold change group $\mathrm{B} / \mathrm{A}$ \\
\hline 219975_x_at & $O L A H$ & Oleoyl-ACP hydrolase & 5.72 \\
\hline 211372_s_at & $I L 1 R 2$ & Interleukin 1 receptor, type II & 4.81 \\
\hline 214637_at & OSM & Oncostatin M & 4.15 \\
\hline 205403_at & $I L 1 R 2$ & Interleukin 1 receptor, type II & 4.11 \\
\hline 224056_at & GNA13 & Guanine nucleotide binding protein ( $\mathrm{G}$ protein) & 4.07 \\
\hline 205110_s_at & $F G F 13$ & Fibroblast growth factor 13 & 4.00 \\
\hline 209960_at & $H G F$ & Hepatocyte growth factor (hepapoietin A; scatter factor) & 3.93 \\
\hline 206208_at & CA4 & Carbonic anhydrase IV & 3.89 \\
\hline 205863_at & S100A12 & S100 calcium binding protein $\mathrm{A} 12$ & 3.76 \\
\hline 206697_s_at & $H P$ & Haptoglobin & 3.41 \\
\hline 208470_s_at & $H P R$ & Haptoglobin-related protein & 3.24 \\
\hline 210610_at & CEACAM1 & carcinoembryonic antigen-related cell adhesion molecule 1 & 3.17 \\
\hline 228824_s_at & $L T B 4 D H$ & Leukotriene B4 12-hydroxydehydrogenase & 3.06 \\
\hline 206950_at & SCN9A & Sodium channel, voltage-gated, type IX, alpha subunit & 3.05 \\
\hline 215838_at & LILRA5 & Leukocyte immunoglobulin-like receptor, subfamily B & 3.01 \\
\hline 217552_x_at & $C R 1$ & Complement component $(3 \mathrm{~b} / 4 \mathrm{~b})$ receptor 1 & 2.98 \\
\hline 206618_at & IL18RI & Interleukin 18 receptor 1 & 2.96 \\
\hline 203936_s_at & $M M P 9$ & Matrix metallopeptidase 9 & 2.86 \\
\hline 206359_at & SOCS3 & Suppressor of cytokine signaling 3 & 2.73 \\
\hline 219669_at & $C D 177$ & Polycythemia rubra vera 1 (PRV1) & 2.72 \\
\hline
\end{tabular}

Table 5. The top 20 most down-regulated types of transcript abundance between pre-and post-treatment

\begin{tabular}{|c|c|c|c|c|c|}
\hline \multirow[b]{2}{*}{ Probe ID } & \multirow[b]{2}{*}{ Gene symbol } & \multirow[b]{2}{*}{ Gene name } & \multicolumn{3}{|c|}{ Fold change pre-/posttreatments } \\
\hline & & & Group A & Group B1 & Group B2 \\
\hline 219669_at & $C D 177$ & Polycythemia rubra vera 1 (PRV1) & 26.55 & 10.48 & 54.34 \\
\hline 153829_at & $M G C 34824$ & Hypothetical protein MGC34824 & 16.13 & 6.90 & 32.67 \\
\hline 216331_at & ITGA7 & Integrin $\alpha 7$ & 20.10 & 4.17 & 27.15 \\
\hline 219975_x_at & $O L A H$ & Oleoyl-ACP hydrolase & 9.916 & 18.25 & 24.60 \\
\hline 219295_s_at & PCOLCE2 & Procollagen C-endopeptidase enhancer 2 & 0.52 & 5.58 & 20.98 \\
\hline 205110_s_at & $F G F 13$ & Fibroblast growth factor 13 & 3.52 & 6.10 & 18.19 \\
\hline 206618_at & ILI8R1 & Interleukin 18 receptor 1 & 6.87 & 5.69 & 18.16 \\
\hline 206950_at & $S C N 9 A$ & Sodium channel, voltage-gated, type IX, $\alpha$ subunit & 3.02 & 3.62 & 17.75 \\
\hline 206359_at & SOCS3 & Suppressor of cytokine signaling 3 & 15.21 & 5.67 & 16.10 \\
\hline 210997_at & $H G F$ & Hepatocyte growth factor & 6.60 & 8.43 & 14.18 \\
\hline 214637_at & $O S M$ & Oncostatin $\mathrm{M}$ & 3.22 & 6.10 & 13.68 \\
\hline 208470_s_at & $H P R$ & Haptoglobin-related protein & 5.44 & 8.94 & 13.62 \\
\hline 210998_s_at & $H G F$ & Hepatocyte growth factor & 3.19 & 3.10 & 12.67 \\
\hline 223767_at & GPR84 & G protein-coupled receptor 84 & 7.14 & 7.92 & 12.44 \\
\hline 217552_x_at & CR1 & Complement component $(3 \mathrm{~b} / 4 \mathrm{~b})$ receptor 1 & 8.49 & 4.47 & 12.38 \\
\hline 206697_s_at & $H P$ & Haptoglobin & 4.45 & 7.99 & 11.84 \\
\hline 207072_at & ILI8RAP & Interleukin 18 receptor accessory protein & 5.25 & 4.83 & 10.93 \\
\hline 227697_at & SOCS3 & Suppressor of cytokine signaling 3 & 8.42 & 4.05 & 10.63 \\
\hline 211372_s_at & $I L 1 R 2$ & Interleukin 1 receptor, type II & 7.60 & 8.51 & 9.93 \\
\hline 209498_at & CEACAM1 & Carcinoembryonic antigen-related cell adhesion molecule 1 & 5.27 & 5.17 & 9.64 \\
\hline
\end{tabular}

(PDGFA), adenosine A3 receptor (ADORA3), integrin $\alpha 9$ (ITGA9), integrin $\beta 2$ (ITGAB2), and inositol 1. 4. 5-triphosphate 3-kinase C (ITPKC).

Agreement of quantitative real-time PCR and the results with microarray expression values for the same transcripts. mRNA levels were measured using quantitative RT-PCR in groups $\mathrm{A}(n=6), \mathrm{B} 1(n=6)$, and $\mathrm{B} 2(n=5)$ to confirm the microarray expression values. Five transcripts were selected that showed the largest difference in the microarray data (PRV-1, HP, CEACAM1, SOCS3, and OSM). The results indicated that the mRNA levels of those transcripts increased in group B in comparison with group A before treatment and most decreased in group B2 after treatment. The results for those transcripts showed markedly significant differences between groups $\mathrm{A}$ and $\mathrm{B}$ before treatment and between groups B1 and B2 after treatment (Fig. 4).

\section{DISCUSSION}

Elevated expression of $m R N A$ related to the IVIG resistant and development CALs in pretreatments samples of group $B$ patients. Similarities in the transcript abundance pattern of groups A, B1, and B2 were investigated before treatment using an unsupervised hierarchical clustering analysis. The results showed more strong similarities between the transcript abundance pattern of groups B1 and B2 than group A $\left(r^{2}=0.56\right.$; Fig. $\left.2 A\right)$. Most of the significantly different transcripts between groups A and B also varied between the IVIG-responsive and IVIG-resistant patients (6316 transcripts). In addition, the more up-regulated transcripts before treatment in group B were related to the development CALs and IVIG resistance $\{$ e.g., acute inflammatory proteins [HP, S100A12], adhesion molecules 


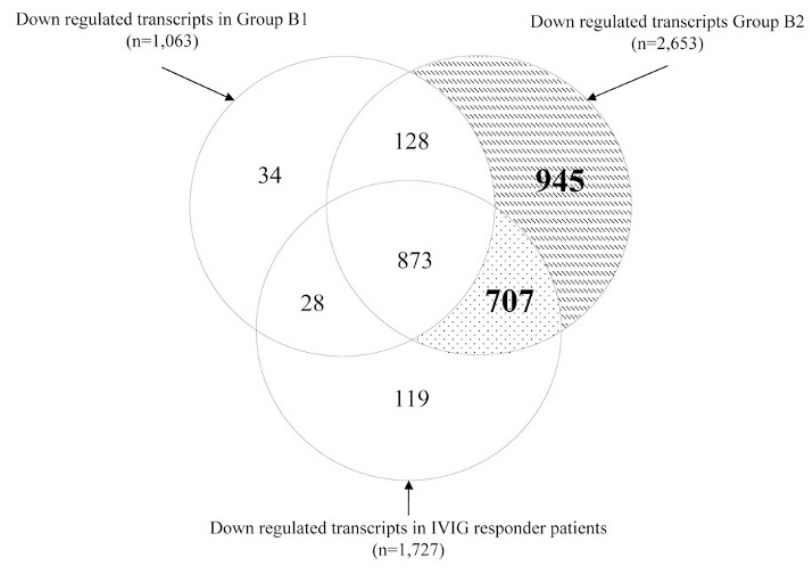

Figure 3. Distribution analysis for the down-regulated transcripts before and after treatment in groups B1 and B2, using a Venn diagram. In this distribution analysis using Venn diagram, there were 707 transcripts that were $\geq 2$ SD down-regulated transcripts before and after treatments in IVIG-responders and group B2 patients, 945 transcripts in only Group B2, 34 transcripts in only group B1 patients (one-way ANOVA: $p<0.05,>2.0 \times$ fold change).

Table 6. Gene ontology classification of the down-regulated transcripts after treatment using GeneSpring GX 7.3

$p$

Categories of down-regulation transcripts in IVIG responsive and group B2 patients (707 transcripts)

Positive regulation of I- $\kappa \mathrm{B}$ kinase/NF- $\kappa \mathrm{B}$ cascade $\quad<0.001$ Immune response

$<0.001$

Regulation of I- $\kappa$ B kinase/NF- $\kappa$ B cascade

$<0.001$

Response to pest, pathogen or parasite

$<0.001$

Apoptosis

$<0.001$

Cauterization in immune response

Positive regulation of cytokine production $\quad<0.001$

Neutrophil activation

$<0.001$

Positive regulation of TNF $\alpha$ biosynthesis $\quad<0.001$

Regulation of cytokine production

0.0012

Positive regulation of IL $1 \beta$ secretion

0.0012

Categories of down-regulation transcripts in only group B2 patients (945 transcripts)

Apoptosis

$<0.001$

Inositol phosphate dephosphorylation

$<0.001$

Ubiquiti cycle

$<0.001$

Protein amino acid dephosphorylation

$<0.001$

Glutathione metabolism

$<0.001$

Cauterization in immune response

B-cell activation

B-cell differentiation

$<0.001$

0.0017

Natural killer cell differentiation

0.0029

Positive regulation of activated T-cell proliferation $\quad 0.028$

Cytotoxic T-cell degranulation

0.044

$p$ value calculation by Fisher's exact test.

[CEACAM1, matrix metallopeptidase 9 (MMP9)], and cell surface molecules [IL1R2, IL18R, PRV-1, CRI]\} (20-25). Therefore, patient stratification according to the Egami score may indeed have reflected the potential responsiveness to IVIG treatment.

The transcripts after the IVIG-plus-IVMP combined therapy were less abundant in the leukocytes than those after the single-IVIG treatment. Although the hierarchical cluster analysis before treatments showed that the transcript abundance pattern of sample from group B1 patients was similar to that

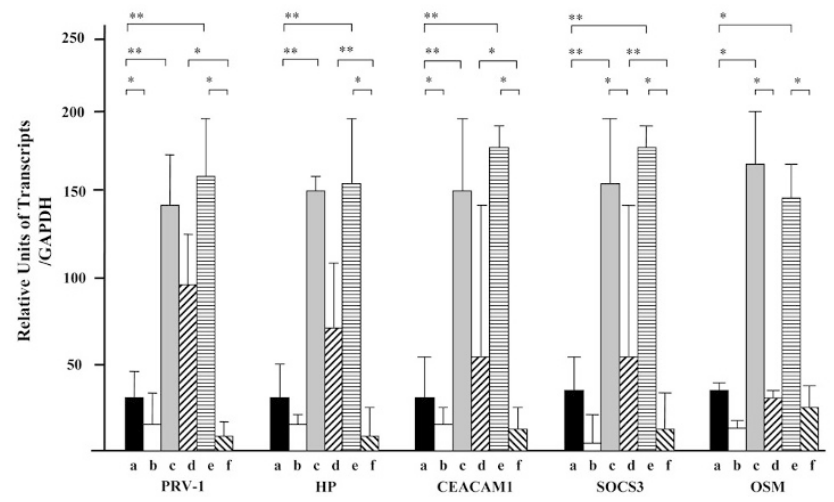

Figure 4. mRNA expression levels in patients before and after treatment in groups A, B1, and B2. The results of quantitative RT-PCR are presented as relative units of each transcript in comparison with GAPDH. a. Group A: pre-IVIG, b. group A: post-IVIG, c. group B1: pre-IVIG, d. group B1: post-IVIG, e. group B2: pre-IVIG + IVMP, f. group B2: post-IVIG + IVMP. Error bars indicate SEM. mRNA levels of those transcripts increased in group $\mathrm{B}$ in comparison with group A before treatment, and most decreased in group B2 after treatments. $* p<0.05, * * p<0.005$ for comparison between pre- and posttreatment results (Wilcoxon signed-rank test) and among groups A, B1, and B2 (Mann-Whitney $U$ test).

of samples from group B2 patients $\left(r^{2}=0.59\right)$. The transcripts abundance pattern after treatments showed strong similarities between IVIG responders $(n=6)$ and group B2 patients $\left(r^{2}=\right.$ 0.66 ; Fig. 2B). This result suggests that administering of combination IVIG-plus-IVMP therapy greatly altered the transcript abundance in the leukocytes of the group B patients. Moreover, group B2 suppressed the largest number of transcripts between the pre- and posttreatment in the three groups (Fig. 3). In our preset study, we did not examine the effects of altering the transcripts after IVMP therapy for the group A patients.

Abe et al. (24) reported the results of a microarray study in which the PRV-1 and HP seemed to be good biomarker for predicting a response to IVIG among patients with KD; these transcripts were correlated with the IVIG response, and higher levels of transcripts of these transcripts were observed in IVIG-resistant patients than in IVIG-responsive patients. A microarray study by Popper et al. (23) showed that the $C E A C A M-1$ is up-regulated in IVIG-resistant patients. In this study, although transcripts such as ILIR2, OSM, HP, CRI, $I L 18 R 1, S O C S 3$ and $P R V-1$, and CEACAM1 were more abundant in group $B$ before treatment, those transcript abundances were suppressed after treatment in each of the groups, and group B2 showed greater down-regulation of those transcripts than groups A and B1 (Tables 4 and 5). The quantitative RT-PCR and the results of the microarray expression values for $P R V-1, H P$, CEACAMI, SOCS3, and OSM were in agreement (Fig. 4). These results suggested that the IVIG-plus-IVMP combined therapy more profoundly and broadly down-regulated the transcripts in the leukocytes than did the single-IVIG treatment.

IVIG-plus-IVMP combination therapy suppressed a wide range of inflammatory cell types in comparison to single IVIG. The Gene Ontology browser analysis showed that the 707 transcripts with down-regulation of $\geq 2 \mathrm{SD}$ in group B2 patients and IVIG responders had functions related to the 
NF- $\kappa \mathrm{B}$ cascade, neutrophil activation, TNF $\alpha$ synthesis, and regulation of IL1 $\beta$. There were 945 transcripts with downregulated of $\geq 2 \mathrm{SD}$ in only group B2 patients had functions related to the apoptosis and to the regulation of $\mathrm{B}$ cells, $\mathrm{T}$ cells, and NK cells (Fig. 3, Table 6). Makata et al. (26) reported that high-dose IVIG inhibits the activation of monocytes/macrophages and coronary arterial endothelial cells more strongly than that of $\mathrm{T}$ cells, whereas dexamethasone inhibits the activation of all three cell types such as monocytes/macrophages, $\mathrm{T}$ cells, and endothelial cells. Although endothelial cells were not analyzed in this study, IVIG-plusIVMP combination therapy also inhibited transcripts associated with a wide range of inflammatory cell types (Fig. 3, Table 6). In addition, it is possible that IVIG-plus-IVMP combined therapy down-regulated the transcript abundance related to neutrophil activation, $\mathrm{TNF} \alpha$ synthesis, and regulation of IL1 $\beta$ (Table 6). The 34 transcripts with downregulation of $\geq 2 \mathrm{SD}$ in only group $\mathrm{B} 1$ included $P D G F A$ as platelet-derived growth factor, ITGA9, ITGB2 as adhesion molecule, and PCGF2, ITPKC as a negative regulator of immune cell activation $(27,28)$. The down-regulation of those transcripts may therefore be related to IVIG-resistant patients. However, there is insufficient evidence for that possibility based on the findings of this study.

This study examined the biologic mechanism of action of IVIG-plus-IVMP combined therapy and single-IVIG using a microarray analysis of samples from patients who had been predicted, according to their Egami score, to be IVIG resistant. The transcripts related to IVIG resistance and to the development of CALs were more abundant in the group $\mathrm{B}$ patients in comparison with those in the group A patients. Moreover, those transcripts in the IVIG-plus-IVMP combined therapy group were more profoundly and broadly suppressed in leukocytes than in the single-IVIG treatment group.

Study limitation. This study, used whole blood samples because it was not possible to extract mRNA from neutrophils. Therefore, any observed differences in transcript abundance before and after treatment may have been influenced by variations between the leukocytes. This study investigated mRNA expression level in leukocytes, but not in endothelial cells, which therefore limits comparisons with previous studies.

The Egami score is highly sensitive and specific in Japanese patients with KD (8). Therefore, it remains unclear whether the Egami score would be equally useful for other populations. Moreover, in part due to the small number of subjects in this study, whether remains uncertain if the development of CALs is a reliable clinical endpoint.

In conclusion, IVIG-plus-IVMP combined therapy was administered as a primary treatment in Japanese patients predicted to be IVIG resistant in a prospective study and investigated the biologic mechanism of IVIG-plus-IVMP combined therapy and single-IVIG treatment for predicting IVIG-resistant patients using a microarray analysis. Pretreatment samples from those patients with an Egami score that predicted IVIG resistance showed an up-regulation of transcripts related to vasculitis and to the development of CALs. Furthermore, the IVIG-plus-IVMP combined ther- apy more profoundly and broadly suppressed the transcript abundance than did the single-IVIG treatment. This study pave the way for a larger clinical trial to test the hypothesis that addition of IVMP to standard therapy may benefit patients predicted to be IVIG-resistant based on the Egami score.

\section{REFERENCES}

1. Kawasaki T, Kosaki F, Okawa S, Shigematsu I, Yanagawa H 1974 A new infantile acute febrile mucocutaneous lymph node syndrome (MLNS) prevailing in Japan. Pediatrics 54:271-276

2. Newburger JW, Takahashi M, Beiser AS, Burns JC, Bastian J, Chung KJ, Colan SD, Duffy CE, Fulton DR, Glode MP, Mason WH, Meissner HC, Rowley AH, Reddy V, Sundel RP, Melish ME, Rosen FS 1991 A single intravenous infusion of gamma globulin as compared with four infusions in the treatment of acute Kawasaki syndrome. N Engl J Med 324:1633-1639

3. Newburger JW, Takahashi M, Gerber MA, Gewitz MH, Tani LY, Burns JC, Shulman ST, Bolger AF, Ferrieri P, Baltimore RS, Wilson WR, Baddour LM, Levison ME, Pallasch TJ, Falace DA, Taubert KA 2004 Diagnosis, treatment, and long-term management of Kawasaki disease: a statement for health professionals from the Committee on Rheumatic Fever, Endocarditis, and Kawasaki Disease, Council on Cardiovascular Disease in the Young, American Heart Association. Pediatrics 114:1708-1733

4. Durongpisitkul K, Gururaj VJ, Park JM, Martin CF 1995 The prevention of coronary artery aneurysm in Kawasaki disease: a meta-analysis on the efficacy of aspirin and immunoglobulin treatment. Pediatrics 96:1057-1061

5. Burns JC, Capparelli EV, Brown JA, Newburger JW, Glode MP 1998 Intravenous gamma-globulin treatment and retreatment in Kawasaki disease. US/Canadian Kawasaki Syndrome Study Group. Pediatr Infect Dis J 17:1144-1148

6. Muta H, Ishii M, Egami K, Furui J, Sugahara Y, Akagi T, Nakamura Y, Yanagawa $\mathrm{H}$, Matsuishi T 2004 Early intravenous gamma-globulin treatment for Kawasaki disease: the nationwide surveys in Japan. J Pediatr 144:496-499

7. Kobayashi T, Inoue Y, Takeuchi K, Okada Y, Tamura K, Tomomasa T, Kobayashi T, Morikawa A 2006 Prediction of intravenous immunoglobulin unresponsiveness in patients with Kawasaki disease. Circulation 113:2606-2612

8. Tremoulet AH, Best BM, Song S, Wang S, Corinaldesi E, Eichenfield JR, Martin DD, Newburger JW, Burns JC 2008 Resistance to intravenous immunoglobulin in children with Kawasaki disease. J Pediatr 153:117-121

9. Egami K, Muta H, Ishii M, Suda K, Sugahara Y, Iemura M, Matsuishi T 2006 Prediction of resistance to intravenous immunoglobulin treatment in patients with Kawasaki disease. J Pediatr 149:237-240

10. Sano T, Kurotobi S, Matsuzaki K, Yamamoto T, Maki I, Miki K, Kogaki S, Hara J 2007 Prediction of non-responsiveness to standard high-dose gamma-globulin therapy in patients with acute Kawasaki disease before starting initial treatment. Eur J Pediatr 166:131-137

11. Okada K, Hara J, Maki I, Miki K, Matsuzaki K, Matsuoka T, Yamamoto T, Nishigaki T, Kurotobi S, Sano T 2009 Pulse methylprednisolone with gamma globulin as an initial treatment for acute Kawasaki disease. Eur J Pediatr 168:181185

12. Wright DA, Newburger JW, Baker A, Sundel RP 1996 Treatment of immune globulin-resistant Kawasaki disease with pulsed doses of corticosteroids. J Pediatr 128:146-149

13. Hashino K, Ishii M, Iemura M, Akagi T, Kato H 2001 Re-treatment for immune globulin-resistant Kawasaki disease: a comparative study of additional immune globulin and steroid pulse therapy. Pediatr Int 43:211-217

14. Furukawa T, Kishiro M, Akimoto K, Nagata S, Shimizu T, Yamashiro Y 2008 Effects of steroid pulse therapy on immunoglobulin-resistant Kawasaki disease. Arch Dis Child 93:142-146

15. Ogata S, Bando Y, Kimura S, Ando H, Nakahata Y, Ogihara Y, Kaneko T, Minoura K, Kaida M, Yokota Y, Furukawa S, Ishii M 2009 The strategy of immune globulin resistant Kawasaki disease: a comparative study of additional immune globulin and steroid pulse therapy. J Cardiol 53:15-19

16. Ayusawa M, Sonobe T, Uemura S, Ogawa S, Nakamura Y, Kiyosawa N, Ishii M, Harada K 2005 Revision of diagnostic guidelines for Kawasaki disease (the 5th revised edition). Pediatr Int 47:232-234

17. Nomura I, Abe J, Noma S, Saito H, Gao B, Wheeler G, Leung DY 2005 Adrenomedullin is highly expressed in blood monocytes associated with acute Kawasaki disease: a microarray gene expression study. Pediatr Res 57: 49-55

18. Abe J, Jibiki T, Noma S, Nakajima T, Saito H, Terai M 2005 Gene expression profiling of the effect of high-dose intravenous Ig in patients with Kawasaki disease. J Immunol 174:5837-5845

19. Livak KJ, Schmittgen TD 2001 Analysis of relative gene expression data using real-time quantitative PCR and the $2-\Delta \Delta C$ T Method. Methods 25:402-408

20. Senzaki H, Masutani S, Kobayashi J, Kobayashi T, Nakano H, Nagasaka H, Sasaki N, Asano H, Kyo S, Yokote Y 2001 Circulating matrix metalloproteinases and their inhibitors in patients with Kawasaki disease. Circulation 104:860-863

21. Foell D, Ichida F, Vogl T, Yu X, Chen R, Miyawaki T, Sorg C, Roth J 2003 S100A12 (EN-RAGE) in monitoring Kawasaki disease. Lancet 361:12701272 
22. Nomura Y, Masuda K, Maeno N, Yoshinaga M, Kawano Y 2004 Serum levels of interleukin-18 are elevated in the subacute phase of Kawasaki syndrome. Int Arch Allergy Immunol 135:161-165

23. Popper SJ, Shimizu C, Shike H, Kanegaye JT, Newburger JW, Sundel RP, Brown PO, Burns JC, Relman DA 2007 Gene-expression patterns reveal underlying biological processes in Kawasaki disease. Genome Biol 8:R261

24. Abe J, Ebata R, Jibiki T, Yasukawa K, Saito H, Terai M 2008 Elevated granulocyte colony-stimulating factor levels predict treatment failure in patients with Kawasaki disease. J Allergy Clin Immunol 122:1008-1013.e1008

25. Takeshita S, Tokutomi T, Kawase H, Nakatani K, Tsujimoto H, Kawamura Y, Sekine I 2001 Elevated serum levels of matrix metalloproteinase-9 (MMP-9) in Kawasaki disease. Clin Exp Immunol 125:340-344
26. Makata H, Ichiyama T, Uchi R, Takekawa T, Matsubara T, Furukawa S 2006 Anti-inflammatory effect of intravenous immunoglobulin in comparison with dexamethasone in vitro: implication for treatment of Kawasaki disease. Naunyn Schmiedebergs Arch Pharmacol 373:325-332

27. Suzuki A, Miyagawa-Tomita S, Komatsu K, Nakazawa M, Fukaya T, Baba K, Yutani C 2004 Immunohistochemical study of apparently intact coronary artery in a child after Kawasaki disease. Pediatr Int 46:590-596

28. Onouchi Y, Gunji T, Burns JC, Shimizu C, Newburger JW, Yashiro M, Nakamura Y, Yanagawa H, Wakui K, Fukushima Y, Kishi F, Hamamoto K, Terai M, Sato Y, Ouchi K, Saji T, Nariai A, Kaburagi Y, Yoshikawa T, Suzuki K, Tanaka T, Nagai T, Cho H, Fujino A, Sekine A, Nakamichi R, Tsunoda T, Kawasaki T, Nakamura Y, Hata A 2008 ITPKC functional polymorphism associated with Kawasaki disease susceptibility and formation of coronary artery aneurysms. Nat Genet 40:35-42 